\title{
Cross-disease analysis identified novel common genes for both lung adenocarcinoma and lung squamous cell carcinoma
}

\author{
GUANGHUI ZHANG ${ }^{1}$, WEIJIE WANG ${ }^{1}$, WEIYANG HUANG ${ }^{1}$, XIAOLI XIE ${ }^{1}$, \\ ZHIGANG LIANG ${ }^{2}$ and $\mathrm{HONGBAO} \mathrm{CAO}^{3}$ \\ ${ }^{1}$ Department of Cardiothoracic Surgery, Ningbo Fourth Hospital, Ningbo, Zhejiang 315037; \\ ${ }^{2}$ Department of Thoracic Surgery, Ningbo First Hospital, Ningbo, Zhejiang 315000, P.R. China; \\ ${ }^{3}$ Statistical Genomics and Data Analysis Core, National Institutes of Health, Bethesda, MD 20852, USA
}

Received September 6, 2018; Accepted May 25, 2019

DOI: $10.3892 / \mathrm{ol} .2019 .10678$

\begin{abstract}
Lung squamous cell carcinoma (LSCC) exhibits a number of similarities with lung adenocarcinoma (LA) in terms of copy number alterations. However, compared with LA, the range of genetic alterations in LSCC is less understood. In the present study, a large-scale literature-based search of LA-associated genes and LSCC-associated genes was performed to identify the genetic basis in common with these two diseases. For each of the LA-associated genes, a mega-analysis was performed to test its expression variations in LSCC using 11 RNA expression datasets, with significant genes identified using statistical analysis. Subsequently, a functional pathway analysis was performed to identify a possible association between any of the significant genes identified from the mega-analysis and LSCC, followed by a co-expression analysis. A multiple linear regression (MLR) model was employed to investigate the possible influence of sample size, country of origin and study date on gene expression in patients with LSCC. Disease-gene association data analysis identified 1,178 genes involved in LA, 334 in LSCC, with a significant overlap of 187 genes $\left(\mathrm{P}<1.02 \mathrm{x}^{-161}\right)$. Mega-analysis revealed that three LA-associated genes, such as solute carrier family 2 member 1 (SLC2A1), endothelial PAS domain protein 1 (EPAS1) and cyclin-dependent kinase 4 (CDK4), were significantly associated with LSCC $\left(\mathrm{P}<1.60 \times 10^{-8}\right)$, with multiple potential pathways identified by functional pathway analysis, which were further validated by co-expression analysis. The present MLR analysis suggested that the country of origin was a significant factor for the levels of expression of all three genes in patients with LSCC $\left(\mathrm{P}<4.0 \times 10^{-3}\right)$. Collectively, the
\end{abstract}

Correspondence to: Dr Zhigang Liang, Department of Thoracic Surgery, Ningbo First Hospital, 59 Liuting Street, Haishu, Ningbo, Zhejiang 315000, P.R. China

E-mail: z.liang@gousinfo.com

Key words: lung adenocarcinoma, lung squamous cell carcinoma, solute carrier family 2 member 1, endothelial PAS domain protein 1, cyclin-dependent kinase 4 present results suggested that genes associated with LA should be further investigated for their association with LSCC. In addition, SLC2A1, EPAS1 and CDK4 may be novel risk genes associated with LA and LSCC.

\section{Introduction}

Non-small-cell lung carcinoma (NSCLC) accounts for $\sim 85 \%$ of all cases of lung cancer worldwide, and the most common histological subtypes of NSCLC are lung adenocarcinoma (LA) and lung squamous cell carcinoma (LSCC) (1). LA and LSCC cells originate from lung epithelial cells and differentiate into glandular and squamous phenotypes, lining the larger airways and the peripheral small airways $(2,3)$. LSCC exhibits many similarities with LA in terms of somatic copy number alterations (4), which raises the possibility of the presence of common genetic features between these two diseases $(5,6)$. Clinical, genetic and biochemical evidence also suggest that different types of lung cancer may share similar molecular pathways (6). However, clinical or pathological phenotypes alone may be insufficient to understand the underlying mechanisms of lung cancer $(5,6)$.

Investigation of disease-associated genes can improve the understanding of disease etiology and development, thereby facilitating design and development of novel preventive and treatment strategies $(7,8)$. Cross disease-gene studies and further pathway analyses provide an opportunity to resolve overlapping associations into discrete pathways and investigate possible shared etiologies $(9,10)$.

The aim of the present study was to identify shared risk genes and to improve the understanding of shared pathways and biological mechanisms involved in LA and LSCC using a mega-analysis of gene expression data. Considering that the range of genetic alterations in LSCC is less understood compared with LA, the present study investigated genes that were involved in LA but not with LSCC using LSCC gene expression datasets.

\section{Materials and methods}

Study design. First, a large-scale literature-based analysis of disease-associated genes was performed to identify genes 
involved in LSCC and LA. Subsequently, for each of the LA-associated genes identified, a mega-analysis was performed using LSCC gene expression data. Pathway analysis was then performed to identify possible functional pathways associated with LSCC-specific genes. Finally, a co-expression-based protein-protein interaction (PPI) analysis was performed using LSCC expression data to evaluate the pathways identified. The workflow diagram is presented in Fig. 1.

LA-and LSCC-associated gene data. LA-and LSCC-associated gene data were acquired from the Pathway Studio (version 12.1.0.9; www.pathwaystudio.com) (11) mammalian database, which is a group of real-time updated literature knowledge databases, including curated signaling pathways, cellular processes, megabolic pathways, ontologies, annotations, molecular interactions and functional associations (http://pathwaystudio.gousinfo.com/ResNetDatabase. $\mathrm{html}$ ). Association data were extracted from $>41,000,000$ references, including PubMed (https://www.ncbi.nlm. nih.gov/pubmed) abstracts and full-text articles. The Pathway database employs an automated natural language processing-based information extraction system, MedScan, with a precision $>91 \%$ (12). Association data within the database are supported with one or more reference. The Pathway Studio ResNet Database is the largest literature database (13). These data were organized into a genetic dataset termed 'LA_LSCC', which is available at the Bioinformatics Database (http://database.gousinfo.com). The downloadable excel spreadsheet containing the dataset is available at http://gousinfo.com/database/Data_Genetic/LA_LSCC.xlsx. The full lists of genes associated with LA and/or LSCC are presented in the groups 'LA_alone genes', 'LSCC_alone genes' and 'Common genes'. In addition, the references for every disease-gene association are presented in the groups 'Ref for LA_alone genes' for LA-specific genes, 'Ref for LSCC_alone genes' for LSCC-specific genes and 'Ref for common genes' for shared genes. Information regarding the titles of the references and the sentences where the disease-gene associations were identified are presented in the 'LA_LSCC' dataset.

Gene expression data selected for mega-analysis. Following the initial search with 'lung squamous cell carcinoma', 158 microarray expression datasets were identified on gene expression omnibus (https://www.ncbi.nlm.nih.gov/geo/) $(14,15)$. Subsequently, the following criteria were applied: i) The organism used in the study was Homo sapiens; ii) the data type was microarray expression profiling; and iii) the studies were limited to comparison between LSCC and healthy controls. A total of 12 datasets satisfied the inclusion criteria for the mega-analysis. However, one dataset (GSE27489 (16); www.ncbi.nlm.nih.gov/geo/query/acc.cgi?acc=GSE27489) was excluded from further investigation as each gene in this dataset demonstrated a small variation in expression level, which may lead to biased results in the mega-analysis. The 11 included datasets are listed in Table I (17-27).

Mega-analysis models. The $\log 2$ fold-change (LFC) of the gene expression level was used to indicate the effect size. Both fixed-effect and random-effects models were employed to investigate and compare the effect size (28). The heterogeneity of the mega-analysis was analyzed to study the variance within and between different studies. In the case that the total variance $(\mathrm{Q})$ was equal to or smaller than the expected between-study variance (df), the within-study variance percentage $(\mathrm{ISq})=100 \% \times(\mathrm{Q}-\mathrm{df}) / \mathrm{Q}$ was set at 0 and a fixed-effect model was selected for the mega-analysis. Otherwise, a random-effects model was selected. Q-p represents the probability that the total variance was only due to within-study variance. Significantly associated genes from this mega-analysis were identified using the following criteria: i) $\mathrm{P}<1 \times 10^{-7}$; and ii) $\mid \mathrm{LFCl}>1$. When a gene exhibited a $\mid \mathrm{LFCl}>1$ in the mega-analysis, the change in the expression level of the gene was $>2$-fold or $<0.5$-fold. The current study presented all the mega-analysis results identified in the 'Mega-analysis' group in the 'LA_LSCC' dataset; however, only genes with a $\mid \mathrm{LFCl}>1$ were further discussed. All analyses were performed using Matlab (version R2017a; https://www.mathworks.com/products/matlab.html).

Multiple linear regression analysis. A multiple linear regression (MLR) model was employed to investigate the possible influence of sample size, country of origin and study date on the gene expression in LSCC. P-values and 95\% CIs were reported for each of these factors.

Pathway analysis. To test the functional profile of the common genes associated with LA and LSCC, a Gene Set Enrichment Analysis (GSEA) was conducted using Pathway Studio (version 12.1.0.9; www.pathwaystudio.com) against Gene Ontology (GO; http://geneontology.org) and Pathway Studio Ontology (version 12.1.0.9; www.pathwaystudio.com). In addition, functional pathway analysis was performed to investigate potential biological associations between the identified risk genes and LSCC. The analysis was performed using the 'Shortest Path' module of Pathway Studio in order to identify various 'entities', including complexes, proteins and functional classes, that were associated to both the genes and LSCC. The reference information included the types of associations, the number of underlying supporting references and the sentences where these associations had been identified and described.

Co-expression analysis. For each pair of the genes and proteins identified in the aforementioned pathway analysis, another mega-analysis was performed to investigate their co-expression using the 11 LSCC expression datasets. The Fisher's Z-value (FisherZ) of Pearson's correlation was used to determine the effect size, and the following equation was used to calculate it: FisherZ $=0.5 \times \log [(1+$ Correlation $)-(1-$ Correlation $)]$. The purpose of this analysis was to validate the associations identified in the pathway analysis. The present study used the following criteria for the selection of a non-random meaningful association: i) An absolute value of FisherZ $>0.3$; and ii) $\mathrm{P}<0.05$. The detailed FisherZ values and P-values are presented in the 'Co-expression' analysis.

\section{Results}

LA and LSCC genes. LA-and LSCC-associated gene analyses revealed 1,178 genes associated with $\mathrm{LA}$, supported by 7,355 
Table I. Datasets used for lung squamous cell carcinoma-gene association mega-analysis.

\begin{tabular}{lcccccc}
\hline Study name & Dataset GEO ID & Control (n) & Case (n) & Study age (years) & Country & (Refs.) \\
\hline Nazarov et al, 2017 & GSE84784 & 9 & 9 & 2 & Luxembourg & $(17)$ \\
Tong et al, 2016 & GSE67061 & 8 & 69 & 3 & China & - \\
Mascaux et al, 2014 & GSE33479 & 27 & 14 & 5 & USA & - \\
Rousseaux et al, 2014 & GSE30219 & 14 & 61 & 5 & France & $(18)$ \\
Girard et al, 2012 & GSE32036 & 59 & 12 & 7 & USA & $(19,20)$ \\
Philipsen et al, 2010 & GSE19188 & 65 & 27 & 9 & Netherlands & $(21)$ \\
Boelens et al, 2009 & GSE12472 & 28 & 35 & 10 & Netherlands & $(22)$ \\
Ishikawa et al, 2009 & GSE2088 & 30 & 48 & 10 & Japan & $(23)$ \\
Boelens et al, 2008 & GSE12428 & 28 & 34 & 11 & Netherlands & $(24)$ \\
Rosskopf et al, 2006 & GSE6044 & 5 & 14 & 13 & Germany & $(25)$ \\
Takeuchi et al, 2009 & GSE11969 & 5 & 35 & 10 & Japan & $(26,27)$ \\
\hline
\end{tabular}

GEO, gene expression omnibus; -, unavailable. Study age =current year -publication year+1.

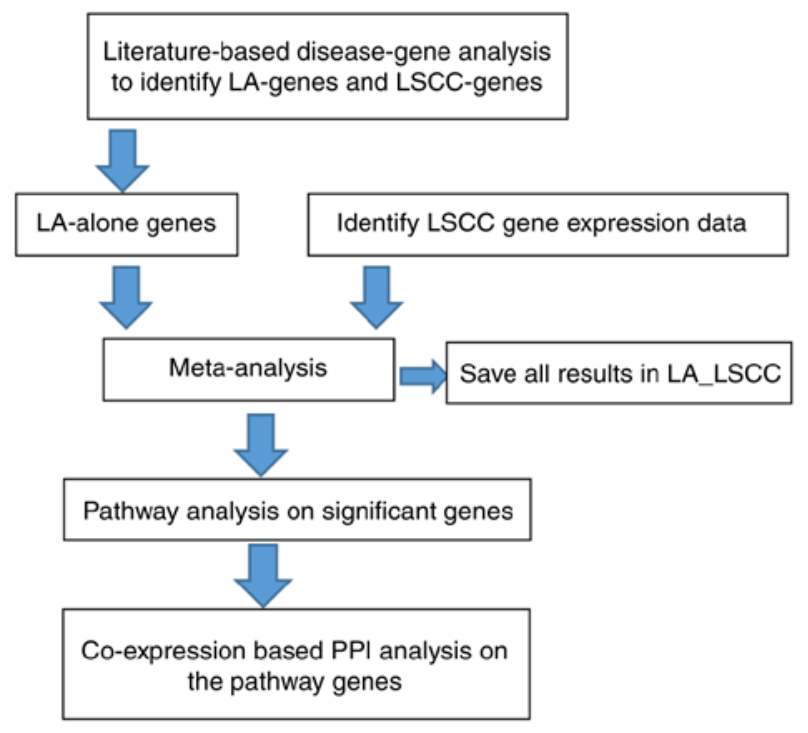

Figure 1. Workflow diagram of the cross LA-LSCC analysis. LA, lung adenocarcinoma; LSCC, lung squamous cell carcinoma; PPI, protein-protein interaction.

references, and 334 genes associated with LSCC, supported by 838 references. The full list of these genes and the associated references are presented in the 'LA_LSCC' dataset. A significant overlap of 187 genes, which are presented in the 'Common genes' group, was identified for both LA and LSCC (right tail Fisher's Exact test; $\mathrm{P}=1.02 \times 10^{-161}$ ). This accounted for $55.99 \%$ of all the LSCC-associated genes and $15.87 \%$ of all the LA-associated genes.

To test the functional profile of the 187 common genes associated with both LA and LSCC, a GSEA was conducted using Pathway Studio against the GO and Pathway Studio Ontology. In total, nine pathways/gene sets (73 unique genes) associated with protein kinase, three pathways/gene sets (71 unique genes) associated with cell growth proliferation, two pathways/gene sets (nine unique genes) associated with cell apoptosis and one pathway/gene set (ten unique genes) associated with transcription factors were significantly enriched. The full list of the 39 pathways/gene sets enriched with $\mathrm{P}<1.7 \times 10^{-5}$ (with 144 out of 187 unique genes) are presented in the 'Common pathways' group contained in the 'LA_LSCC' dataset. The majority of these pathways were involved in LA and LSCC, indicating a shared genetic basis for these diseases.

Three novel common genes in LA and LSCC. Although an overlap was identified between LA-and LSCC-associated genes, the majority of the LA-specific genes (991 genes, $84.13 \%$ ) were not implicated in LSCC. A systematic mega-analysis was performed to collectively assess differential expressed mRNAs and determine whether previously investigated LA-associated genes were also linked to LSCC. Notably, certain datasets do not contain the three genes and therefore will not be included in the current study. However, the LFCs of the genes were estimated from the majority of the 11 studies ( $>9$ studies). The associations between the LA-specific genes with 11 LSCC gene expression datasets (Table I) were evaluated. A total of three genes, including solute carrier family 2 member 1 (SLC2A1), endothelial PAS domain protein 1 (EPAS1) and cyclin-dependent kinase 4 (CDK4), passed the significant criteria $\left(\mathrm{P}<1 \times 10^{-7}\right.$ and $\left.\mathrm{ILFCl}>1\right)$ and are presented in Table II. The detailed results are presented in the 'Mega-analysis' group in the 'LA_LSCC' dataset.

The effect sizes, 95\% CIs and weights of different studies for the three identified genes (SLC2A1, EPAS1 and CDK4) are presented in Fig. 2. EPAS1 and CDK4 exhibited significant variances between studies ( ISq $>0 \%$ and $\mathrm{Q}$-test $\mathrm{P}<0.1$ ). Therefore, the random-effects model was selected for their mega-analysis. By contrast, no significant between-study variance was observed for SLC2A1 (Q-test P>0.4), and the fixed-effect model was selected for SLC2A1 (Fig. 2). Notably, multiple line regression analyses demonstrated that the country of origin was a significant factor that influenced the LFC of all three genes in the case of LSCC $(\mathrm{P}<0.004$; Table II) 
Table II. Statistically significant genes identified from the mega-analysis of lung squamous cell carcinoma.

\begin{tabular}{|c|c|c|c|c|c|c|c|c|}
\hline \multirow{3}{*}{$\begin{array}{l}\text { Gene } \\
\text { name }\end{array}$} & \multirow{3}{*}{$\begin{array}{c}\text { Random effects } \\
\text { model }\end{array}$} & \multirow{3}{*}{$\begin{array}{c}\text { Datasets } \\
\text { included (n) }\end{array}$} & \multirow{2}{*}{\multicolumn{3}{|c|}{ Mega-analysis results }} & \multicolumn{3}{|c|}{ MLR analysis results (P-values) } \\
\hline & & & & & & \multirow{2}{*}{$\begin{array}{l}\text { Sample } \\
\text { size }\end{array}$} & \multirow[b]{2}{*}{$\begin{array}{l}\text { Population } \\
\text { region }\end{array}$} & \multirow[b]{2}{*}{$\begin{array}{l}\text { Study } \\
\text { age }\end{array}$} \\
\hline & & & LFC & SD of LFC & P-value & & & \\
\hline SLC2A1 & 0 & 11 & 1.63 & 0.25 & $4.31 \times 10^{-11}$ & 0.59 & $4 \times 10^{-3}$ & 0.69 \\
\hline EPAS1 & 1 & 9 & -1.50 & 0.26 & $1.27 \times 10^{-8}$ & 0.49 & $2 \times 10^{-5}$ & 0.09 \\
\hline CDK4 & 1 & 11 & 1.02 & 0.18 & $1.60 \times 10^{-8}$ & 0.87 & $6 \times 10^{-5}$ & 0.98 \\
\hline
\end{tabular}

SLC2A1, solute carrier family 2 member 1; EPAS1, endothelial PAS domain protein 1; CDK4, cyclin-dependent kinase 4; LFC, log-fold change; SD, standard deviation. D Study age =current year -publication year+1.
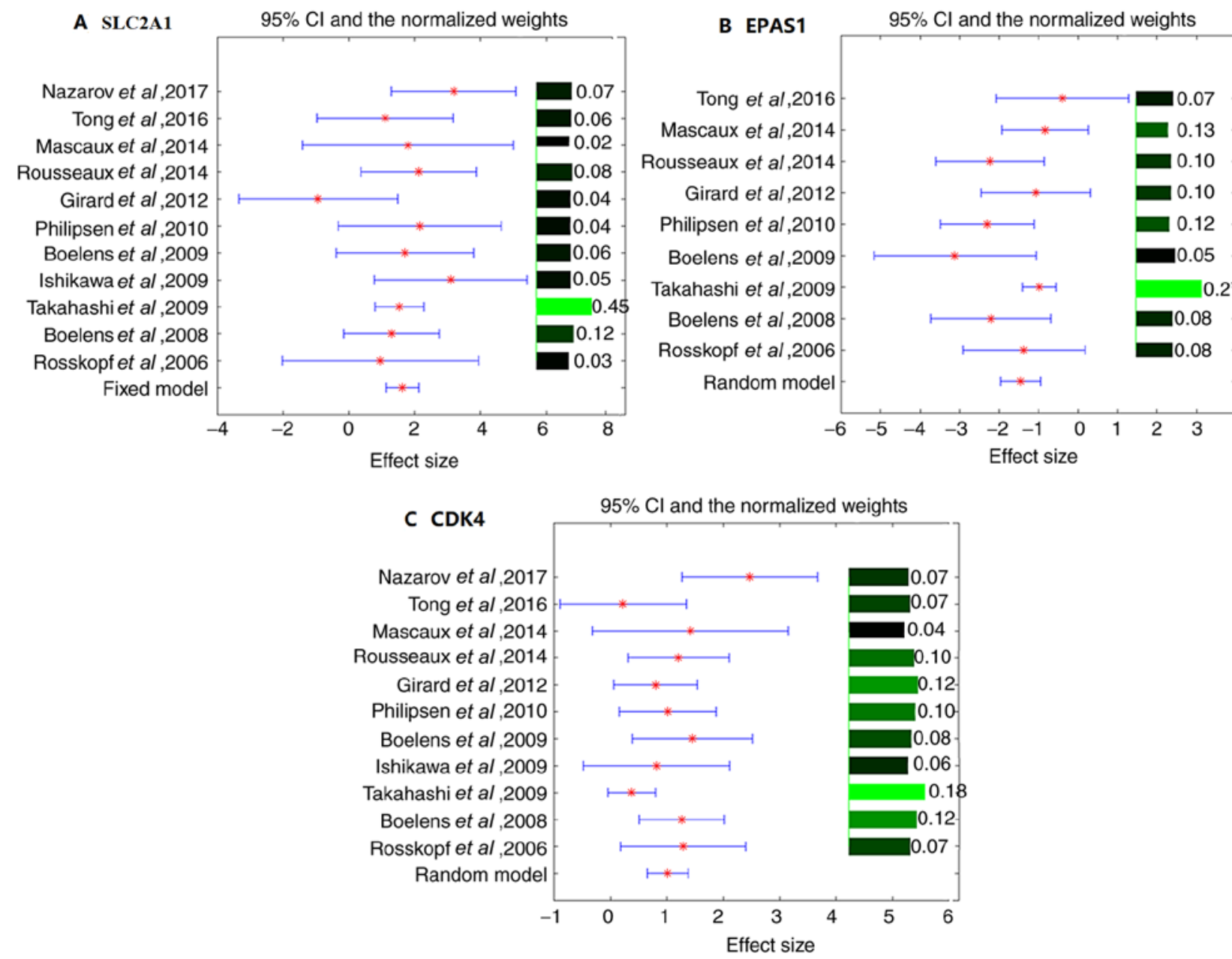

Figure 2. Effect size, 95\% CI and weights for the genes SLC2A1, EPAS1, and CDK4. Results from a mega-analysis using (A) a fix-effect model for SLC2A1, and a random-effects model for (B) EPAS1 and (C) CDK4. SLC2A1, solute carrier family 2 member 1; EPAS1, endothelial PAS domain protein 1; CDK4, cyclin-dependent kinase 4.

Functional pathway analysis. According to the approach used to identify the genes associated with LSCC, SLC2A1, EPAS1 and CDK4 exhibited no direct link with LSCC. However, functional pathway analysis revealed multiple potential pathways through which these three genes may serve roles in the pathology of LSCC (Fig. 3). Each edge in Fig. 3 was supported by $\geq 1$ references, and details of these associations are presented in the 'LSCC-3Genes_potential pathways' group in the 'LA_LSCC' dataset.
To confirm the associations presented in Fig. 3, a co-expression PPI analysis was conducted with the purpose of validating the associations between CD4K4, EPAS1 and SLC2A1, and the 13 other genes presented in Fig. 3. The majority of the entities presented in Fig. 3 also exhibited significant associations in the co-expression analysis (Fig. 4), supporting the pathway analysis results. Co-expression analysis results are presented in the 'Co-expression' group in the 'LA_LSCC' dataset. 


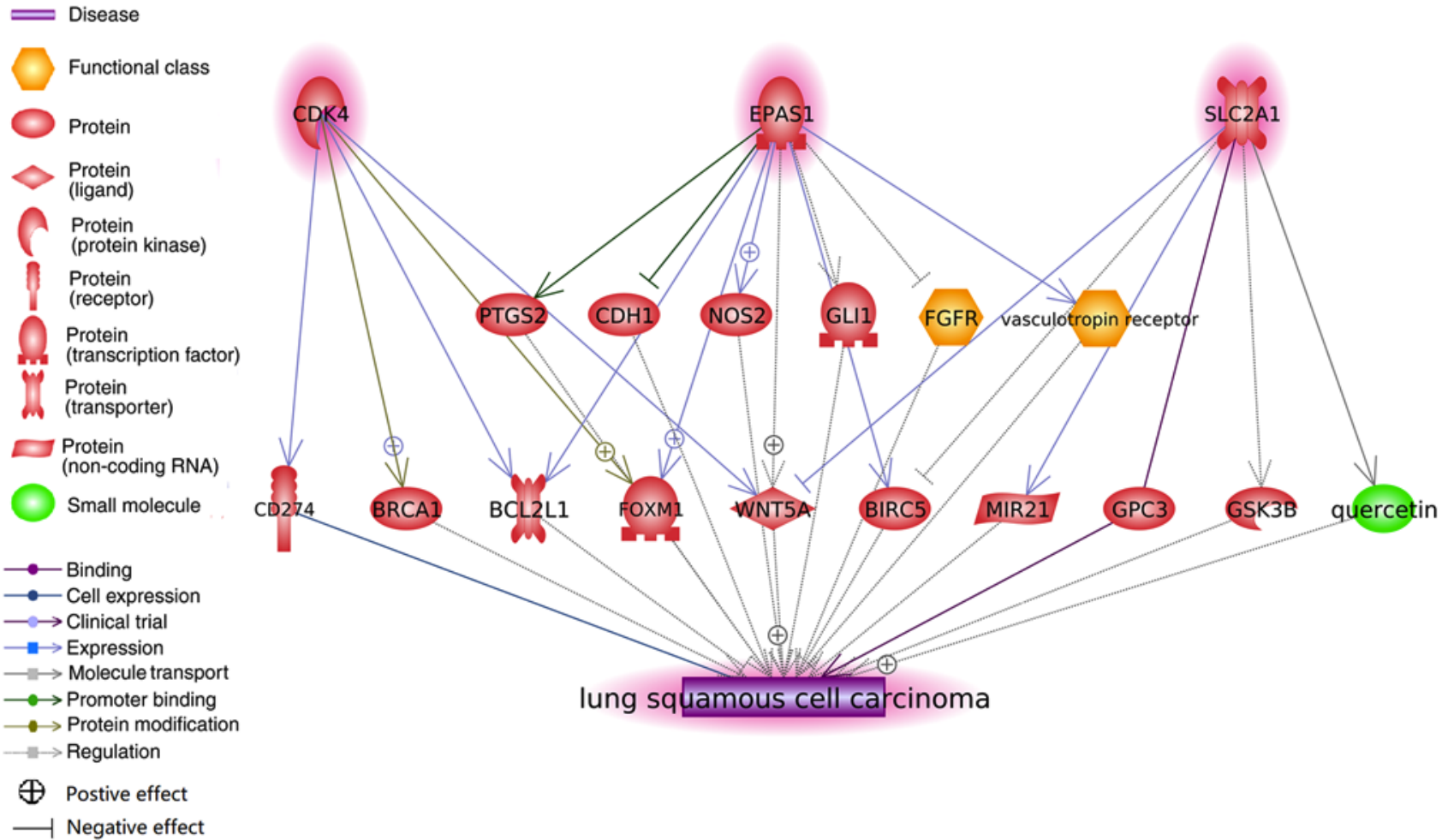

Figure 3. Potential pathways associating SLC2A1, EPAS1 and CDK4 to lung squamous cell carcinoma. Network was generated using Pathway Studio. Each association (edge) has $\geq 1$ supporting reference. SLC2A1, solute carrier family 2 member 1; EPAS1, endothelial PAS domain protein 1; CDK4, cyclin-dependent kinase 4; PTGS2, prostaglandin-endoperoxide synthase 2; CDH1, cadherin 1; NOS2, nitric oxide synthase 2; GLI1, GLI family member zinc finger 1; FGFR, fibroblast growth factor receptor; BCL2L1, BCL2-like 1; FOXM1, forkhead box M1; WNT5A, Wnt family member 5A; BIRC5, baculoviral IAP repeat containing 5; MIR21, microRNA-21; GPC3, glypican 3; GSK3B, glycogen synthase kinase $3 \beta$.

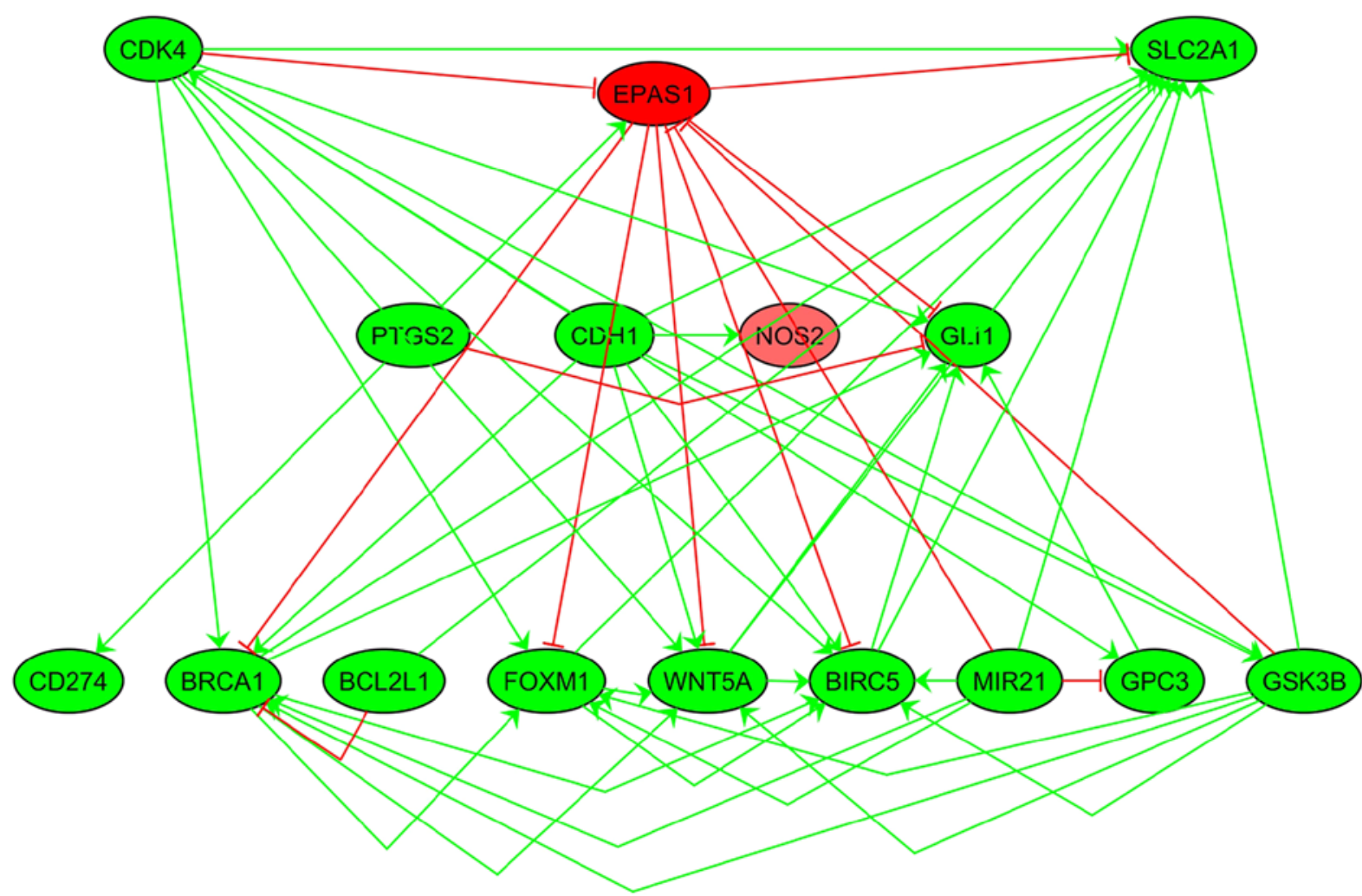

Figure 4. Co-expression analysis. Each edge represents a significant association between the corresponding two entities $(\mathrm{P}<0.05)$. Positive associations are highlighted in green and negative associations are highlighted in red. Nodes in red indicate decreased expression and green nodes indicate increased expression. SLC2A1, solute carrier family 2 member 1; EPAS1, endothelial PAS domain protein 1; CDK4, cyclin-dependent kinase 4; PTGS2, prostaglandin-endoperoxide synthase 2; CDH1, cadherin 1; NOS2, nitric oxide synthase 2; GLI1, GLI family member zinc finger 1; BCL2L1, BCL2-like 1; FOXM1, forkhead box M1; CD274, cluster of differentiation 274; WNT5A, Wnt family member 5A; BIRC5, baculoviral IAP repeat containing 5; MIR21, microRNA-21; GPC3, glypican 3 . 


\section{Discussion}

The cross-analysis of different lung cancer phenotypes may facilitate the development of novel strategies and approaches for the treatment of lung cancer. In the present study, LA-specific genes were systematically mega-analyzed with LSCC differential expression data and three genes, including SLC2A1, EPAS1 CDK4, were identified as potential risk genes for LSCC. Importantly, whether these associations between the genes and LSCC indicate causality requires further investigation.

It is a major concern that a disease-gene association derived from experiment-based literature is heavily dependent on the quality and access of the text data, and the efficiency of the mining algorithms. Candidate disease-gene analysis is more appropriate for monogenic diseases because the association between genotype and phenotype is clearer $(11,12)$. In lung cancer, a complex disease, the etiology can be attributed to tobacco smoking, sex, ethnicity, age, diet, obesity, infections and numerous genes that work in combination to elicit the disease phenotype (29-31). It has also been observed that when individually investigated, the genes potentially responsible for the disease may not result in disease in certain patients (32-35).

In this context, cross-disease analysis based on mega-analysis can overcome the limitations of sample size and identify more reliable and robust common genes between LA and LSCC through the quantitative combination and assessment of multiple studies $(36,37)$. In the present study, disease-gene association data were retrieved from the Pathway Studio database and mega-analysis was performed to detect their significance in terms of gene expression levels. All of these analyses can provide a more reliable and robust result.

The present study used MLR analysis to demonstrate that lung cancer outcome varies among different populations and ethnicities. In addition, the present study identified that the country of origin may be associated with the expression levels of SLC2A1, EPAS1 and CDK4 in the case of LSCC. It is therefore necessary to assess the generalizability of the present results in different ethnic groups. Socioeconomic and cultural differences among different racial groups may account for some degree of the current disparities and a personalized molecular approach may help to resolve such problems (38-41).

The current literature-based functional pathway analysis revealed several possible pathways that link the three novel genes identified to LSCC. For example, CDK4, a member of the serine/threonine protein kinase family, may contribute to the development of LSCC via a CDK4-forkhead box M1 (FOXM1)-LSCC pathway. It has been reported that CDK4 activity can increase the transcriptional activity of FOXM1 without phosphorylating FOXM1 (42), while the expression of FOXM1 has been suggested to contribute to the development or progression of LSCC (43). A previous study also suggested that CDK4 can stimulate the BRCA1 promoter in an E2F transcription factor 1-dependent manner, regulating cell cycle, DNA replication and cell proliferation processes (44). BRCA1 serves an important role in LSCC via cell cycle and DNA replication signaling pathways (44), which indicates a potential CDK4-BRCA1-LSCC pathway.

EPAS1 can bind to and inhibit the expression of the calcium-dependent cell adhesion molecule cadherin 1 (CDH1) $(45,46)$. CDH1 has been reported to serve a dual role in the maintenance of the LSCC phenotype (47). These previous studies indicate that the EPAS1-CDH1-LSCC pathway may serve a complex role in LSCC development. EPAS1 regulates the production of prostaglandin-endoperoxide synthase (PTGS) (48), which has been indicated to promote the carcinogenesis of LSCC, suggesting a potential EPAS1-PTGS-LSCC pathway.

SLC2A1 is a major glucose transporter responsible for constitutive or basal glucose uptake, which can bind with glypican 3 (GPC3) to decrease glucose transport activity (49) and transport quercetin to balance the glucose efflux (50). Mechanisms associated with glucose efflux are also identified in the pathological process of $\operatorname{LSCC}(51,52)$, suggesting the existence of SLC2A1-GPC3-LSCC and SLC2A1-quercetin-LSCC pathways.

Co-expression analysis revealed that the majority of the identified genes were associated with each other in terms of expression. The majority of the literature-based pathway identified was validated by the expression data-based associations found. However, a certain number of the associations identified in the present study may not be consistent with the present co-expression analysis. For example, SLC2A1 inhibits the expression of baculoviral IAP repeat containing 5 (BIRC5) in the pathway analysis; however, SLC2A1 and BIRC5 exhibit positive co-expression in the co-expression analysis, which indicates the presence of a more complex genetic network including more regulators.

The present cross-disease analysis between LA and LSCC suggested common genes may contribute to disease comorbidities and trait manifestations. The novel common genes identified may facilitate the development of novel strategies targeting shared mechanisms across diseases. However, the conclusion of the current study was only based on a statistical analysis of previous experimental data and a literature-based pathway study. Therefore, further biological experiments, including gene-knockout or knockdown experiments, are required to validate the associations between the three genes identified and LSCC.

In conclusion, cross-disease analysis could provide a powerful tool to investigate new targets and reveal common biological mechanisms. Genes associated with LA require further analysis to identify their association with LSCC. SLC2A1, EPAS1 and CDK4 genes identified in the present study may be novel common risk genes associated with both LA and LSCC.

\section{Acknowledgements}

Not applicable.

\section{Funding}

No funding was received.

\section{Availability of data and materials}

The datasets generated and/or analyzed during the current study are available in the http://database.gousinfo.com repository, http://gousinfo.com/database/Data_Genetic/LA_LSCC. xlsx. 


\section{Authors' contributions}

GZ and ZL developed the study design, supervised the whole the study process and prepared the manuscript. WW and $\mathrm{HC}$ contributed to data analysis and manuscript drafting and revision. WH and XX contributed to data collection and manuscript drafting and revision. All authors approve the final version of the manuscript.

\section{Ethics approval and consent to participate}

Not applicable.

\section{Patient consent for publication}

Not applicable.

\section{Competing interests}

The authors declare that they have no competing interests.

\section{References}

1. Ansari J, Shackelford RE and El-Osta H: Epigenetics in non-small cell lung cancer: From basics to therapeutics. Transl Lung Cancer Res 5: 155-171, 2016.

2. Catacchio I, Scattone A, Silvestris N and Mangia A: Immune prophets of lung cancer: The prognostic and predictive landscape of cellular and molecular immune markers Transl Oncol 11: $825-835,2018$

3. Chalela R, Curull V, Enríquez C, Pijuan L, Bellosillo B and Gea J: Lung adenocarcinoma: From molecular basis to genome-guided therapy and immunotherapy. J Thorac Dis 9: 2142-2158, 2017.

4. Cancer Genome Atlas Research Network: Comprehensive genomic characterization of squamous cell lung cancers. Nature 489: 519-525, 2012.

5. Hirsch FR, Spreafico A, Novello S, Wood MD, Simms L and Papotti M: The prognostic and predictive role of histology in advanced non-small cell lung cancer: A literature review. J Thorac Oncol 3: 1468-1481, 2008.

6. Pankratz VS, Sun Z, Aakre J, Li Y, Johnson C, Garces YI, Aubry MC, Molina JR, Wigle DA and Yang P: Systematic evaluation of genetic variants in three biological pathways on patient survival in low stage non-small cell lung cancer. J Thorac Oncol 6: 1488-1495, 2011.

7. Yandell M, Huff C, Hu H, Singleton M, Moore B, Xing J, Jorde LB and Reese MG: A probabilistic disease-gene finder for personal genomes. Genome Res 21: 1529-1542, 2011.

8. Zhang Y, Shen F, Mojarad MR, Li D, Liu S, Tao C, Yu Y and Liu H: Systematic identification of latent disease-gene associations from PubMed articles. PLoS One 13: e0191568, 2018.

9. Ellinghaus D, Jostins L, Spain SL, Cortes A, Bethune J, Han B, Park YR, Raychaudhuri S, Pouget JG, Hübenthal M, et al: Analysis of five chronic inflammatory diseases identifies 27 new associations and highlights disease-specific patterns at shared loci. Nature Genetics 48: 510-518, 2016.

10. Chen D, Che N, Le J and Pan QL: A co-training based entity recognition approach for cross-disease clinical documents. Con Comput Pract Exp: e4505, 2018.

11. Nikitin A, Egorov S, Daraselia N and Mazo I: Pathway studio-the analysis and navigation of molecular networks. Bioinformatics 19 : 2155-2157, 2003.

12. Daraselia N, Yuryev A, Egorov S, Novichkova S, Nikitin A and Mazo I: Extracting human protein interactions from MEDLINE using a full-sentence parser.Bioinformatics 20: 604-611, 2004.

13. Lorenzi PL, Claerhout S, Mills GB and Weinstein JN: 'A curated census of autophagy-modulating proteins and small molecules: Candidate targets for cancer therapy'. Autophagy 10: 1316-1326, 2014.

14. Edgar R, Domrachev M and Lash AE: Gene expression omnibus: NCBI gene expression and hybridization array data repository. Nucleic Acids Res 30: 207-210, 2002.
15. Barrett T, Wilhite SE, Ledoux P, Evangelista C, Kim IF, Tomashevsky M, Marshall KA, Phillippy KH, Sherman PM, Holko M, et al: NCBI GEO: Archive for functional genomics data sets-update. Nucleic Acids Res 41 (Database Issue): D991-D995, 2013.

16. Kahn N, Meister M, Eberhardt R, Muley T, Schnabel PA, Bender C, Johannes M, Keitel D, Sültmann H, Herth FJ, et al: Early detection of lung cancer by molecular markers in endobronchial epithelial-lining fluid. J Thorac Oncol 7: 1001-1008, 2012.

17. Nazarov PV, Muller A, Kaoma T, Nicot N, Maximo C, Birembaut P, Tran NL, Dittmar G and Vallar L: RNA sequencing and transcriptome arrays analyses show opposing results for alternative splicing in patient derived samples. BMC Genomics 18: 443, 2017.

18. Rousseaux S, Debernardi A, Jacquiau B, Vitte AL, Vesin A, Nagy-Mignotte H, Moro-Sibilot D, Brichon PY, Lantuejoul S, Hainaut $\mathrm{P}$, et al: Ectopic activation of germline and placental genes identifies aggressive metastasis-prone lung cancers. Sci Transl Med 5: 186ra66, 2013.

19. Byers LA, Diao L, Wang J, Saintigny P, Girard L, Peyton M, Shen L, Fan Y, Giri U, Tumula PK, et al: An epithelial-mesenchymal transition gene signature predicts resistance to EGFR and PI3K inhibitors and identifies Axl as a therapeutic target for overcoming EGFR inhibitor resistance. Clin Cancer Res 19: 279-290, 2013.

20. Schuster K, Venkateswaran N, Rabellino A, Girard L, Peña-Llopis S and Scaglioni PP: Nullifying the CDKN2AB locus promotes mutant K-ras lung tumorigenesis. Mol Cancer Res 12: 912-923, 2014.

21. Hou J, Aerts J, den Hamer B, van Ijcken W, den Bakker M, Riegman P, van der Leest C, van der Spek P, Foekens JA, Hoogsteden HC, et al: Gene expression-based classification of non-small cell lung carcinomas and survival prediction. PLoS One 5: e10312, 2010.

22. Boelens MC, Gustafson AM, Postma DS, Kok K, van der Vries G, van der Vlies P, Spira A, Lenburg ME, Geerlings M, Sietsma H, et al: A chronic obstructive pulmonary disease related signature in squamous cell lung cancer. Lung Cancer 72: 177-183, 2011.

23. Fujiwara T, Hiramatsu M, Isagawa $T$, Ninomiya $H$, Inamura $\mathrm{K}$, Ishikawa S, Ushijima M, Matsuura M, Jones MH, Shimane M, et al: ASCL1-coexpression profiling but not single gene expression profiling defines lung adenocarcinomas of neuroendocrine nature with poor prognosis. Lung Cancer 75: 119-125, 2012.

24. Boelens MC, van den Berg A, Fehrmann RS, Geerlings M, de Jong WK, te Meerman GJ, Sietsma H, Timens W, Postma DS and Groen HJ: Current smoking-specific gene expression signature in normal bronchial epithelium is enhanced in squamous cell lung cancer. J Pathol 218: 182-191, 2009.

25. Rohrbeck A, Neukirchen J, Rosskopf M, Pardillos GG, Geddert H, Schwalen A, Gabbert HE, von Haeseler A, Pitschke G, Schott M, et al: Gene expression profiling for molecular distinction and characterization of laser captured primary lung cancers. J Transl Med 6: 69, 2008.

26. Takeuchi T, Tomida S, Yatabe Y, Kosaka T, Osada H, Yanagisawa K, Mitsudomi $\mathrm{T}$ and Takahashi T: Expression profile-defined classification of lung adenocarcinoma shows close relationship with underlying major genetic changes and clinicopathologic behaviors. J Clin Oncol 24: 1679-1688, 2006.

27. Matsuyama Y, Suzuki M, Arima C, Huang QM, Tomida S, Takeuchi T, Sugiyama R, Itoh Y, Yatabe Y, Goto $H$ and Takahashi T: Proteasomal non-catalytic subunit PSMD2 as a potential therapeutic target in association with various clinicopathologic features in lung adenocarcinomas. Mol Carcinog 50: 301-309, 2011.

28. Borenstein M, Hedges LV, Higgins JP and Rothstein HR: A basic introduction to fixed-effect and random-effects models for mega-analysis. Res Synth Methods 1: 97-111, 2010.

29. Davila DG and Williams DE: The etiology of lung cancer. Mayo Clin Proc 68: 170-182, 1993.

30. Williams MD and Sandler AB: The epidemiology of lung cancer. Cancer Treat Res 105: 31-52, 2001.

31. Lemjabbar-Alaoui H, Hassan OU, Yang YW and Buchanan P: Lung cancer: Biology and treatment options. BBA-Reviews on Cancer 1856: 189-210, 2015.

32. Piro RM and Di Cunto F: Computational approaches to disease-gene prediction: Rationale, classification and successes. FEBS J 279: 678-696, 2012

33. Opap K and Mulder N: Recent advances in predicting gene-disease associations. F1000Res 6: 578, 2017. 
34. Doncheva NT, Kacprowski T and Albrecht M: Recent approaches to the prioritization of candidate disease genes. Wiley Interdiscip Rev Syst Biol Med 4: 429-442, 2012.

35. Lee TI and Young RA: Transcriptional regulation and its misregulation in disease. Cell 152: 1237-1251, 2013.

36. Botling J, Edlund K, Lohr M, Hellwig B, Holmberg L, Lambe M, Berglund A, Ekman S, Bergqvist M, Pontén F, et al: Biomarker discovery in non-small cell lung cancer: Integrating gene expression profiling, mega-analysis and tissue microarray validation. Clin Cancer Res 19: 194-204, 2013.

37. Ramasamy A, Mondry A, Holmes CC and Altman DG: Key issues in conducting a mega-analysis of gene expression microarray datasets. PLoS Med 5: e184, 2008.

38. El-Telbany A and Ma PC: Cancer genes in lung cancer: Racial disparities: Are there any? Genes Cancer 3: 467-480, 2012.

39. Siegel R, Ward E, Brawley O and Jemal A: Cancer statistics, 2011: The impact of eliminating socioeconomic and racial disparities on premature cancer deaths. Ca Cancer J Clin 61 212-236, 2011

40. Schabath MB, Cress D and Munoz-Antonia T: Racial and ethnic differences in the epidemiology and genomics of lung cancer. Cancer Control 23: 338-346, 2016.

41. Hardy D, Liu CC, Xia R, Cormier JN, Chan W, White A, Burau K and Du XL: Racial disparities and treatment trends in a large cohort of elderly black and white patients with nonsmall cell lung cancer. Cancer 115: 2199-2211, 2009.

42. Wierstra I: CyclinD1/Cdk4 increases the transcriptional activity of FOXM1c without phosphorylating FOXM1c. Biochem Biophys Res Commun 431: 753-759, 2013.

43. Yang DK, Son CH, Lee SK, Choi PJ, Lee KE and Roh MS Forkhead box M1 expression in pulmonary squamous cell carcinoma: Correlation with clinicopathologic features and its prognostic significance. Hum Pathol 40: 464-470, 2009.

44. Zhang F, Chen X, Wei K, Liu D, Xu X, Zhang X and Shi H: Identification of key transcription factors associated with lung squamous cell carcinoma. Med Sci Monit 23: 172-206, 2017.
45. Maru S, Ishigaki Y, Shinohara N, Takata T, Tomosugi N and Nonomura K: Inhibition of mTORC2 but not mTORC1 up-regulates E-cadherin expression and inhibits cell motility by blocking HIF-2 $\alpha$ expression in human renal cell carcinoma. J Urol 189: 1921-1929, 2013.

46. Murugesan T, Rajajeyabalachandran G, Kumar S, Nagaraju S and Jegatheesan SK: Targeting HIF-2 $\alpha$ as therapy for advanced cancers. Drug Discov Today 23: 1444-1451, 2018.

47. Pallier K, Cazes A, El Khattabi L, Lecchi C, Desroches M, Danel C, Riquet M, Fabre-Guillevin E, Laurent-Puig P and Blons H: DeltaN TP63 reactivation, epithelial phenotype maintenance, and survival in lung squamous cell carcinoma. Tumor Biol 33: 41-51, 2012.

48. Xiong J, Zhu FF and Nie MF: Hypoxia-inducible factor- $2 \alpha$ (HIF-2 $\alpha$ ) mediates the effects of hypoxia on the promotion of HeLa cell viability, colony formation, and invasion capacity in vitro. Genet Mol Res 14: 3281-3292, 2015.

49. Cho HS, Ahn JM, Han HJ and Cho JY: Glypican 3 binds to GLUT1 and decreases glucose transport activity in hepatocellular carcinoma cells. J Cell Biochem 111: 1252-1292, 2010.

50. Cunningham P, Afzal-Ahmed I and Naftalin RJ: Docking studies show that d-glucose and quercetin slide through the transporter GLUT1. J Biol Chem 281: 5797-5803, 2006.

51. Li K, Pan X, Bi Y, Xu W, Chen C, Gao H, Shi B, Jiang H, Yang S, Jiang L and Li Z: Adoptive immunotherapy using T lymphocytes redirected to glypican-3 for the treatment of lung squamous cell carcinoma. Oncotarget 7: 2496-2507, 2016.

52. Yang JH, Hsia TC, Kuo HM, Chao PD, Chou CC, Wei YH and Chung JG: Inhibition of lung cancer cell growth by quercetin glucuronides via G2/M arrest and induction of apoptosis. Drug Metab Dispos 34: 296-304, 2006.

This work is licensed under a Creative Commons Attribution-NonCommercial-NoDerivatives 4.0 International (CC BY-NC-ND 4.0) License. 